\title{
CXCL5 stimulation of RANK ligand expression in Paget's disease of bone
}

\author{
Kumaran Sundaram¹, D Sudhaker Rao², William L Ries ${ }^{3}$ and Sakamuri V Reddy ${ }^{1}$
}

Paget's disease of bone (PDB) is a chronic focal skeletal disorder that affects $2-3 \%$ of the population over 55 years of age. PDB is marked by highly localized areas of bone turnover with increased osteoclast activity. Evidence suggests a functional role for measles virus nucleocapsid protein (MVNP) in the pathogenesis of PDB. In the present study, we identified elevated levels ( $\sim 180$-fold) of CXCL5 mRNA expression in bone marrow cells from patients with PDB compared with that in normal subjects. In addition, CXCL5 levels are increased (five-fold) in serum samples from patients with PDB. Furthermore, MVNP transduction in human bone marrow monocytes significantly increased CXCL5 mRNA expression. Realtime PCR analysis showed that CXCL5 stimulation increased (6.8-fold) RANKL mRNA expression in normal human bone marrow-derived stromal (SAKA-T) cells. Moreover, CXCL5 increased (5.2-fold) CXCR1 receptor expression in these cells. We further showed that CXCL5 treatment elevated the expression levels of phospho-ERK1/2 and phospho-p38. CXCL5 also significantly increased phosphorylation of CREB (CAMP response element-binding) in bone marrow stromal/preosteoblast cells. Chromatin immuneprecipitation (ChIP) assay confirmed phospho-CREB binding to RANKL gene promoter region. Further, the suppression of p-CREB expression by the inhibitors of ERK1/2, p38 and PKA significantly decreased CXCL5 stimulation of hRANKL gene promoter activity. Thus, our results suggest that CREB is a downstream effector of CXCL5 signaling and that increased levels of CXCL5 contribute to enhanced levels of RANKL expression in PDB.

Laboratory Investigation (2013) 93, 472-479; doi:10.1038/labinvest.2013.5; published online 25 February 2013

KEYWORDS: CXCL5; measles virus nucleocapsid protein (MVNP); osteoclast; Paget's disease of bone; RANK ligand

Paget's disease of bone (PDB) is characterized by highly localized areas of bone turnover with increased osteoclast (OCL) activity followed by poor quality new bone formation due to an exaggerated osteoblast response. Approximately $1 \%$ of patients with PDB develop osteosarcoma. ${ }^{1} \mathrm{PDB}$ is inherited as an autosomal dominant trait with genetic heterogeneity and affects $2-3 \%$ of the population over the age of 55 years. Both genetic and environmental factors are involved in the pathogenesis of PDB. Mutations in the ubiquitin-associated domain of sequestosome 1 (SQSTM1/p62) have been identified at 40\% in familial and $10 \%$ in sporadic cases. ${ }^{2}$ Environmental factors such as paramyxoviruses are implicated in PDB. ${ }^{3}$ We have previously detected the expression of measles virus nucleocapsid (MVNP) transcripts in OCL from patients with PDB. ${ }^{4}$ Moreover, the expression of MVNP in OCL precursors develops pagetic OCL in mice. ${ }^{5}$

Receptor activator for nuclear factor- $\kappa \mathrm{B}$ ligand (RANKL) is expressed by marrow stromal/osteoblast cells in response to several osteotropic factors such as 1,25 dihydroxy vitamin $D_{3}$, parathyroid hormone $(\mathrm{PTH})$, prostaglandin $\mathrm{E}_{2}$ and interleukin $1 \beta$ (IL-1 $\beta) .{ }^{6-8}$ RANKL-RANK signaling is critical for osteoclast differentiation/bone resorption activity. Enhanced levels of RANKL are associated with PDB. ${ }^{9,10}$ In addition, elevated levels of IL-6, macrophage colony-stimulating factor (M-CSF) and endothelin-1 have been reported in PDB. ${ }^{11,12}$ Recently, we showed fibroblast growth factor-2 (FGF-2) levels are increased in patients with PDB and induce RANKL expression. ${ }^{13}$ However, molecular mechanisms underlying the increased levels of RANKL expression in PDB are unclear.

Chemokines are a superfamily of small cytokine-like proteins involved in angiogenesis, cellular growth, cellular motility and inflammatory response. ${ }^{14,15}$ CXCL5 (also known as epithelial neutrophil activating factor-78 (ENA-78)) is a member of CXC chemokine family secreted by neutrophils, monocytes, macrophage, fibroblast and endothelial cells. ${ }^{16}$ CXCL5 binds with CXCR1 and CXCR2 and activates MAP kinase and PI3 kinase signaling pathways. ${ }^{17}$ It has been reported that CXCL5 levels are significantly increased in

${ }^{1}$ Charles P Darby Children's Research Institute, Charleston, SC, USA; ${ }^{2}$ Henry Ford Hospital, Detroit, MI, USA and ${ }^{3}$ College of Dental Medicine, Medical University of South Carolina, Charleston, SC, USA

Correspondence: Dr SV Reddy, PhD, Charles P Darby Children's Research Institute, Medical University of South Carolina, 173 Ashley Avenue, Charleston 29425 SC USA. E-mail: reddysv@musc.edu 
rheumatoid arthritis. ${ }^{18}$ Moreover, inflammatory cytokine IL$1 \beta$ has been shown to induce CXCL5 expression by activation of NF- $\kappa$ B and CREB. ${ }^{19}$ Stromal cell-derived factor-1 (SDF-1 or CXCL12) is a chemokine highly expressed by bone endothelium, bone marrow stromal cells and osteoblast cells. ${ }^{20}$ SDF-1 stimulation of MMP-9 has an important role in osteoclast differentiation/bone resorption activity. ${ }^{21}$ Macrophage inflammatory protein (MIP)- $1 \alpha$ and $\beta$ produced by myeloma cells induce RANKL expression in stromal cells. ${ }^{22}$ Recently, we reported that CXCL13 stimulates RANKL expression in bone marrow stromal/preosteoblast cells. ${ }^{23}$ In the present study, we demonstrated that increased levels of CXCL5 contribute to enhanced levels of RANKL expression in $\mathrm{PDB}$.

\section{MATERIALS AND METHODS Reagents and Antibodies}

Cell culture and DNA transfection reagents were purchased from Invitrogen (Carlsbad, CA, USA). Human recombinant CXCL5, anti-CXCL5 and anti-human RANKL antibody were obtained from R\&D systems (Minneapolis, MN, USA). Antiphospho-CREB, phospho-ERK1/2 and p-38 antibodies were purchased from Cell Signaling (Danvers, MA, USA). Peroxidase-conjugated secondary antibodies were purchased from Santa Cruz Biotechnology (Santa Cruz, CA, USA). Supersignal-enhanced chemiluminescence (ECL) reagent was obtained from Amersham Bioscience (Piscataway, NJ, USA), and nitrocellulose membranes were purchased from Millipore (Bedford, MA, USA). A luciferase reporter assay system was obtained from Promega (Madison, WI, USA). Chromatin Immunoprecipitation (ChIP) assay kit was purchased from Upstate (Temecula, CA, USA). ERK1/2 activation inhibitor (PD98059) and p38 MAPK inhibitor (SB203580) were obtained from Calbiochem (La Jolla, CA, USA).

\section{MVNP Retroviral Expression}

We have previously developed a retroviral plasmid construct, pLXSN, that transcribes MVNP mRNA expression under the control of $5^{\prime}$-LTR viral promoter elements. The recombinant plasmid construct was transfected into the PT67 amphotropic packaging cell line using lipofectamine (Invitrogen). Stable clonal cell lines producing MVNP recombinant retrovirus at high titer $\left(1 \times 10^{6}\right.$ virus particles $\left./ \mathrm{ml}\right)$ were established by selecting for resistance to neomycin $(600 \mu \mathrm{g} / \mathrm{ml})$. Similarly, a control retrovirus producer cell line was established by transfecting the cells with the pLXSN empty vector (EV). Retroviral supernatants from the producer cell cultures were collected and filtered $(0.45 \mu \mathrm{m}$ pore diameter) for immediate use. Human bone marrow-derived mononuclear cells were transduced with EV or MVNP retroviral supernatants (20\%) in the presence of polybrene $(4 \mu \mathrm{g} / \mathrm{ml})$ for $48 \mathrm{~h} .{ }^{4}$

\section{Enzyme-Linked Immunosorbent Assay (ELISA)}

CXCL5 in serum samples from age-matched normal subjects, patients with PDB (mean age $70 \pm 10$ ) and conditioned media (CM) collected from MVNP-transduced human bone marrow-derived mononuclear cells was assayed by ELISA using anti-CXCL5 antibody. All the PDB patients had either mono- or polyostotic with elevated levels of alkaline phosphatase activity. All human samples were obtained following the IRB-approved protocol at the Medical University of South Carolina. Total protein content of the samples was determined by BCA protein assay kit (Pierce, Rockford, IL, USA). CXCL5 levels as measured by ELISA were normalized with the total protein content in all samples analyzed.

\section{Quantitative Real-Time PCR}

RANKL, CXCR1 and -2 mRNA expressions in bone marrow stromal/preosteoblast cells were measured by real-time PCR as described previously. ${ }^{24}$ Briefly, total RNA was isolated from human bone marrow stromal/preosteoblast cells stimulated with and without CXCL5 $(0-50 \mathrm{ng} / \mathrm{ml})$ for $48 \mathrm{~h}$, using RNAzol reagent (Biotecx Labs, Houston, TX, USA). A reverse transcription reaction was performed using poly-dT primer and Moloney murine leukemia virus reverse transcriptase (Applied Biosystem) in a $25 \mu \mathrm{l}$ reaction volume containing total RNA $(2 \mu \mathrm{g}), 1 \times$ PCR buffer and $2 \mathrm{mM} \mathrm{MgCl}_{2}$, at $42^{\circ} \mathrm{C}$ for $15 \mathrm{~min}$ followed by $95^{\circ} \mathrm{C}$ for $5 \mathrm{~min}$. The quantitative real-time PCR was performed using IQ SYBR Green Supermix in an iCycler (iCycler iQ Single-color Real Time PCR detection system; Bio-Rad, Hercules, CA, USA). The primer sequences used to amplify glyceraldehyde3-phosphate dehydrogenase (GAPDH) mRNA were $5^{\prime}$ CCTACCCCCAATGTATCCGTTGTG-3' (sense) and $5^{\prime}$-GG AGGAATGGGAGTTGCTGTTGAA-3' (anti-sense); hRANKL mRNA were $5^{\prime}$-ACCAGCATCAAAATCCCAAG-3' (sense) and $5^{\prime}$-TAAGGAGTTGGAGACCT-3' (anti-sense); hCXCR1 mRNA were $5^{\prime}$-TTTGTTTGTCTTGGCTGCTG-3' (sense) and $5^{\prime}$-TTTCCCAGGACCTCATGACA-3' (anti-sense) and hCXCR2 mRNA were $5^{\prime}$-TCTCACTCCTGAAGGAAGTCA AC- $3^{\prime}$ (sense) and $5^{\prime}$-AGTAGCGCTTCTGGGTCAGT- $3^{\prime}$ (antisense). Thermal cycling parameters were $94^{\circ} \mathrm{C}$ for $3 \mathrm{~min}$, followed by 40 cycles of amplifications at $94^{\circ} \mathrm{C}$ for $30 \mathrm{~s}, 60^{\circ} \mathrm{C}$ for $1 \mathrm{~min}, 72^{\circ} \mathrm{C}$ for $1 \mathrm{~min}$, and $72^{\circ} \mathrm{C}$ for $5 \mathrm{~min}$ as the final elongation step. Relative levels of mRNA expression were normalized in all the samples analyzed with respect to GAPDH amplification.

\section{hRANKL Promoter-Luciferase Reporter Gene Assay}

We have previously developed the hRANKL gene promoter (from +1 to $-2 \mathrm{~kb}$ relative to the transcription start site as per Genbank Accession no. AF 333234)-luciferase reporter plasmid construct (hRANKL P\#3) as described. ${ }^{25}$ Normal human bone marrow derived SAKA-T stromal cells were cultured in $\alpha$-Minimum Essential Medium (MEM) supplemented with $10 \%$ fetal bovine serum, 100 units per $\mathrm{ml}$ of penicillin/streptomycin. DNA transfections were performed using lipofectamine reagent (Invitrogen). Cells were transiently transfected with hRANKL P\#3 plasmid. Cells were cultured in the presence or absence of CXCL5 

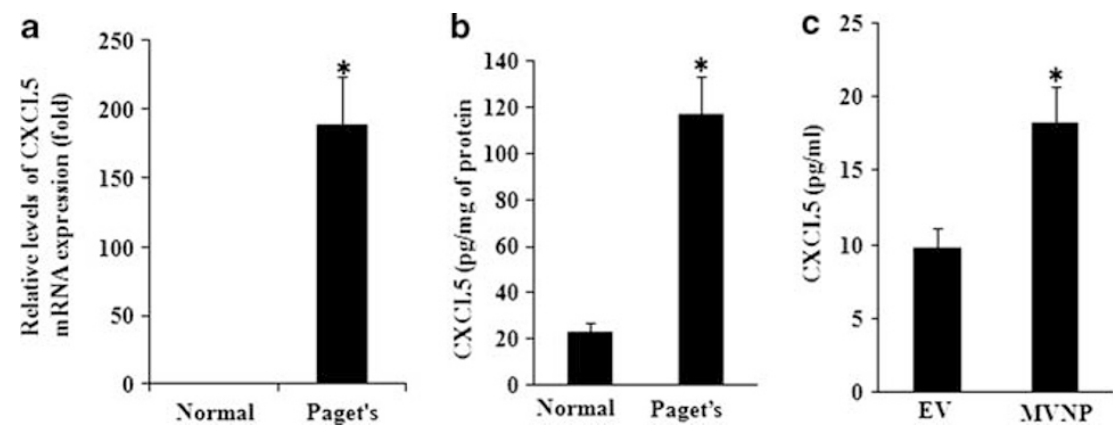

Figure 1 CXCL5 expression in Paget's disease of bone (PDB). (a). Total RNA isolated from normal and PDB patients $(n=7)$ bone marrow monocytes was subjected to real-time PCR analysis for CXCL5 mRNA expression. (b). CXCL5 levels in serum samples from normal subjects and patients with PDB $(n=10)$. (c). Serum-free conditioned media (CM) was collected from human bone marrow-derived monocytes transduced with control empty vector (EV) or MVNP retrovirus expression vectors and stimulated with RANKL $(100 \mathrm{ng} / \mathrm{ml})$ and M-CSF $(10 \mathrm{ng} / \mathrm{ml})$ for $48 \mathrm{~h}$. CXCL5 levels were measured by ELISA. Data represent triplicate studies and are shown as mean \pm s.d. $\left({ }^{*} P<0.05\right)$.

(50 ng/ml) for $48 \mathrm{~h}$. A $20 \mu \mathrm{l}$ aliquot of total cell lysates was mixed with $100 \mu \mathrm{l}$ of the luciferase assay reagent. The light emission was measured for $10 \mathrm{~s}$ of integrated time using Sirius Luminometer following the manufacturer's instructions (Promega). The transfection efficiency was normalized by cotransfection with pRSV $\beta$-gal plasmid and measuring the $\beta$-galactosidase activity in these cells (Promega).

\section{Chromatin Immuneprecipitation (ChIP) Assay}

Human bone marrow-derived stromal cells (SAKA-T) were treated with or without CXCL5 $(50 \mathrm{ng} / \mathrm{ml})$ for $24 \mathrm{~h}$. Cells were crosslinked with $1 \%$ formaldehyde at $37^{\circ} \mathrm{C}$ for $10 \mathrm{~min}$. Crude nuclei were collected by centrifugation at $4000 \times \mathrm{g}$ for $3 \mathrm{~min}$ and resuspended in SDS lysis buffer. The samples were subjected to ChIP assay. Briefly, soluble chromatin was prepared by sonication with a Branson-250 digital sonifier (Branson Ultrasonics, Danbury, CT, USA) to an average DNA length of 200-1000 bp. Approximately $5 \times 10^{5}$ cell equivalents (1/6th) of the sheared soluble chromatin was precleared with blocked Protein G agarose, and 10\% of the precleared chromatin was set aside for input as a positive control. Samples were immunoprecipitated overnight at $4{ }^{\circ} \mathrm{C}$ using anti-p-CREB antibody or equivalent concentrations of normal rabbit IgG as a negative control. Immune complexes were pulled down using Protein $G$ agarose, washed and eluted twice with $250 \mu \mathrm{l}$ of elution buffer $\left(0.1 \mathrm{M} \mathrm{NaHCO}_{3}\right.$, $1 \%$ SDS) and crosslinking reversed in $200 \mathrm{mM} \mathrm{NaCl}$ at $65^{\circ} \mathrm{C}$ overnight with $20 \mu \mathrm{g}$ RNase A. To analyze the CREB-binding region to the RANKL promoter, immunoprecipitated chromatin DNA samples were amplified by PCR using primer pairs for the CREB-binding region ( -1590 to $-1583 \mathrm{bp}$ ) in the RANKL promoter $5^{\prime}$-GGTCAAAGACTACAAGGAGTA- $3^{\prime}$ (sense) and 5'-CAGCCCATACAAAAGAAAATGATCT- $3^{\prime}$ (anti-sense). Immuneprecipitated DNA samples or input DNA fractions were analyzed by real-time PCR analysis as described above. Thermal cycling parameters were 40 cycles of $94{ }^{\circ} \mathrm{C}$ for $30 \mathrm{~s}, 55^{\circ} \mathrm{C}$ for $30 \mathrm{~s}$, and $72{ }^{\circ} \mathrm{C}$ for $30 \mathrm{~s}$ with a final extension at $72{ }^{\circ} \mathrm{C}$ for $5 \mathrm{~min}$. The percentage of chromatinimmunoprecipitated DNA relative to input was calculated and shown as mean \pm s.d. from three independent experiments. PCR products were subjected to electrophoresis by using 3\% agarose gels and visualized by ethidium bromide.

\section{Statistical Analysis}

Results are presented as mean \pm s.d. for three independent experiments and were compared by Student's $t$-test. Values were considered significantly different for $P<0.05$.

\section{RESULTS}

\section{CXCL5 Levels are Elevated in Patients with PDB}

Paget's disease of bone is marked by enhanced levels of RANKL. Chemokines have an important role in pleotropic cellular functions and gene expression. However, chemokine expression and contribution to increased levels of RANKL expression in PDB is unknown. In the present study, we analyzed the levels of CXCL5 mRNA expression in patients with PDB. Total RNA isolated from normal and pagetic sitesderived bone marrow monocytes was subjected to real-time PCR analysis for CXCL5 mRNA expression. We identified that CXCL5 mRNA expression was significantly increased (180-fold) in patients with PDB compared with that in normal subjects (Figure 1a). Further, we measured the CXCL5 levels in serum samples obtained from patients with PDB. ELISA analysis revealed that CXCL5 levels are elevated (five-fold) in PDB patients compared with that in normal subjects (Figure 1b).

We previously reported the expression of measles virus nucleocapsid (MVNP) transcripts in bone marrow cells and peripheral blood-derived monocytes from patients with PDB. ${ }^{26}$ Further, MVNP has been shown to stimulate pagetic OCL development. ${ }^{5}$ We therefore analyzed CXCL5 levels in conditioned medium (CM) obtained from MVNPtransduced normal human bone marrow monocytes by ELISA. As shown in Figure 1c, CM obtained from MVNPtransduced cells demonstrated a significant increase in the levels of CXCL5 compared with empty vector (EV)-transduced cells. These results suggest that CXCL5 levels are elevated in PDB. 

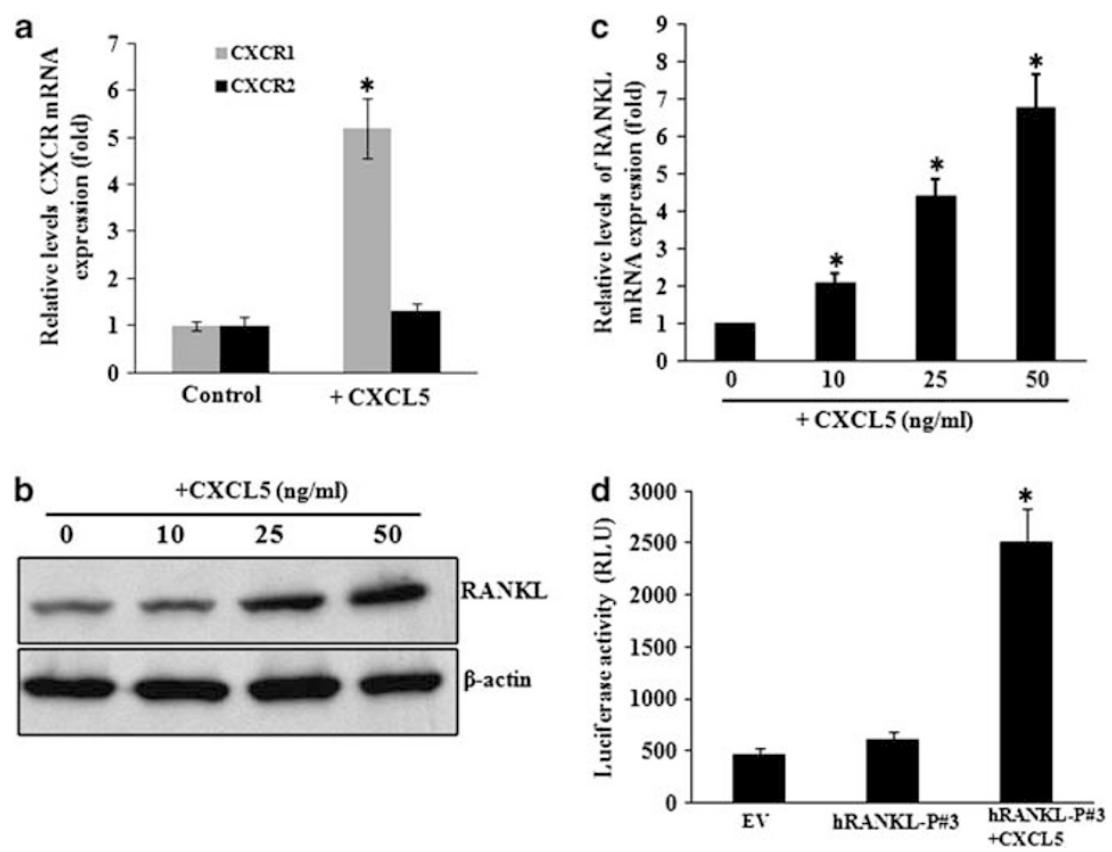

Figure 2 CXCL5 stimulates RANKL expression in human bone marrow-derived stromal/preosteoblast cells. (a). Human bone marrow-derived stromal/ preosteoblast cells were stimulated with CXCL5 $(50 \mathrm{ng} / \mathrm{ml})$ for $48 \mathrm{~h}$. Total RNA isolated was subjected to real-time PCR analysis for CXCR1 and -2 mRNA expressions. (b-c). Cells were stimulated with different concentrations of CXCL5 $(0-50 \mathrm{ng} / \mathrm{ml})$ for $48 \mathrm{~h}$. Total cell lysates obtained were subjected to western blot analysis for RANKL expression and total RNA isolated was subjected to real-time PCR analysis for RANKL mRNA expression. The relative levels of mRNA expression in the samples were normalized by GAPDH amplification. (d). Human bone marrow-derived SAKA-T stromal cells were transfected with empty vector (EV), hRANKL-Luc reporter plasmid (hRANKL P\#3) and stimulated with CXCL5 (50 ng/ml) for $48 \mathrm{~h}$. Total cell lysates were assayed for luciferase activity. The transfection efficiency was normalized by $\beta$-galactosidase activity coexpressed in these cells. Data represent triplicate studies and are shown as mean \pm s.d. $\left({ }^{*} P<0.05\right)$.

\section{CXCL5 Stimulates RANKL Expression in Bone Marrow Stromal/Preosteoblast Cells}

It has been shown that CXCL5 interacts with CXCR1 and CXCR2 receptors on the cell surface. ${ }^{27}$ Therefore, we examined CXCL5 modulation of CXCR1 and -2 mRNA expression in normal human bone marrow-derived stromal/ preosteoblast cells. Cells were stimulated with CXCL5 (50 ng/ $\mathrm{ml}$ ) for $48 \mathrm{~h}$ and total RNA isolated was subjected to realtime PCR analysis for CXCR1 and -2 mRNA expression. We showed that CXCL5 increased (5.2-fold) the level of CXCR1 mRNA expression and in contrast, there was no change in the level of CXCR2 mRNA expression in these cells (Figure 2a). We next determined whether CXCL5 regulates RANKL expression in human bone marrow-derived stromal/preosteoblast cells. Normal human bone marrow-derived stromal/preosteoblast cells were stimulated with different concentrations of CXCL5 $(0-50 \mathrm{ng} / \mathrm{ml})$ for $48 \mathrm{~h}$. Total cell lysates obtained were subjected to western blot analysis for RANKL expression. As shown in Figure 2b, CXCL5 stimulation significantly increased RANKL expression in a dosedependent manner. Real-time PCR analysis of total RNA isolated from these cells demonstrated elevated levels of RANKL mRNA expression (Figure 2c). We further examined the functional role of CXCL5 in the transcriptional regulation of hRANKL gene promoter activity in normal human bone marrow-derived SAKA-T stromal cells. hRANKL promoter- luciferase reporter plasmid was transiently transfected into SAKA-T-cells using lipofectamine. Cells were cultured in the presence or absence of CXCL5 $(50 \mathrm{ng} / \mathrm{ml})$ for $48 \mathrm{~h}$. Total cell lysates obtained from these cells were analyzed for luciferase activity as described. We thus identified that CXCL5 stimulation resulted in a significant increase (five-fold) in hRANKL gene promoter activity compared with unstimulated cells (Figure 2d). These results suggest that CXCL5 modulates CXCR1 expression and may have a functional role in enhanced levels of RANKL expression in stromal/preosteoblast cells. Taken together, these results indicate a functional role for CXCL5 in enhanced levels of RANKL expression associated with PDB.

\section{CXCL5 Signaling in Stromal/Preosteoblast Cells}

We further examined CXCL5 signaling mechanisms to induce RANKL expression in human bone marrow stromal/preosteoblast cells. Normal primary human bone marrow-derived stromal/preosteoblast cells were stimulated with CXCL5 (50 ng/ $\mathrm{ml})$ for different time points $(0-3 \mathrm{~h})$. Western blot analysis of total cell lysates revealed that CXCL5 increased phospho-ERK1/ 2, phospho-p38 and p-CREB expression. However, there was no change in ERK1/2, p38 and CREB expression in these cells (Figure 3). It has been reported that p-ERK1/2 and p-p38 MAP kinases activate downstream signaling molecules such as CREB (cAMP response element-binding). ${ }^{28}$ Therefore, our results 


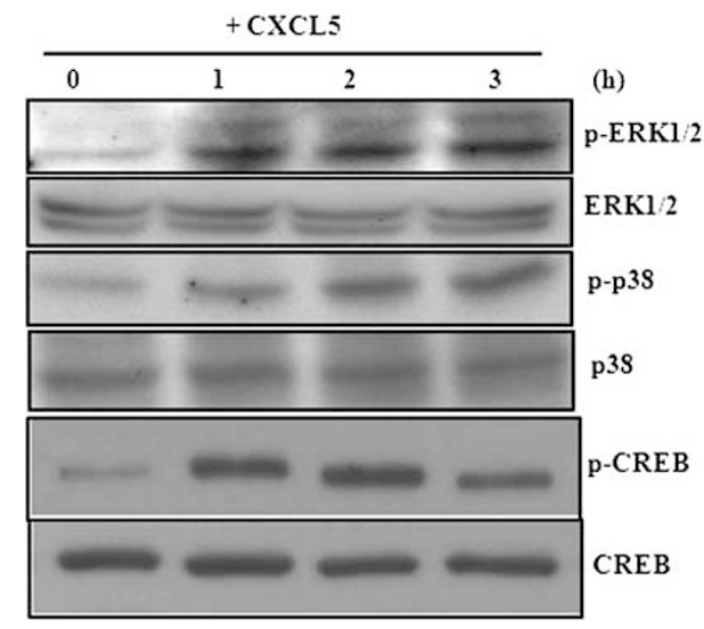

Figure 3 CXCL5 signaling in stromal/preosteoblast cells. Primary human bone marrow-derived stromal/preosteoblast cells were stimulated with CXCL5 $(50 \mathrm{ng} / \mathrm{ml})$ for the indicated time points $(0-3 \mathrm{~h})$. Total cell lysates were subjected to western blot analysis for total and p-ERK1/2, p-p38 and $\mathrm{p}$-CREB expression.

suggests that p-CREB is a downstream effector of CXCL5 signaling to stimulate RANKL expression in bone marrow stromal/preosteoblast cells.

\section{Participation of p-CREB in CXCL5-induced RANKL Expression}

We previously cloned and characterized the human RANKL gene promoter region. ${ }^{25}$ We identified a putative CREBbinding element $(-1590$ to $-1583 \mathrm{bp})$ in the hRANKL promoter region by web-based TFSEARCH analysis. Therefore, we further confirmed p-CREB binding to the hRANKL gene promoter element by ChIP assay using anti-pCREB antibody as described. Chromatin immune complexes obtained from CXCL5-stimulated SAKA-T stromal cells were analyzed by PCR using hRANKL gene-specific primers for the CREB-binding region. As shown in Figures $4 \mathrm{a}$ and $\mathrm{b}$, CXCL5 significantly stimulated p-CREB binding to the RANKL promoter region. A low level of p-CREB-binding at basal level could be due to a transformed phenotype of the SAKA-T cells. We have detected no amplification using primer sets specific for an additional segment in the hRANKL promoter region that lacks a CREB-binding element in the absence or presence of CXCL5 stimuli (data not shown). We then examined the potential of p-CREB to regulate RANKL gene promoter activity in response to CXCL5 stimulation of SAKA-T stromal cells. We used protein kinase A (PKA) inhibitor H89 for inhibition of p-CREB in these cells. Cells were transfected with empty vector (EV), hRANKL gene promoter-luc plasmid construct and stimulated with CXCL5 $(50 \mathrm{ng} / \mathrm{ml})$ in the presence or absence of H89 $(1 \mu \mathrm{M})$ for $48 \mathrm{~h}$. The PKA inhibitor was added $30 \mathrm{~min}$ prior to CXCL5 stimulation and the inhibition of $\mathrm{p}$-CREB expression was measured at the time points indicated. Total cell lysates
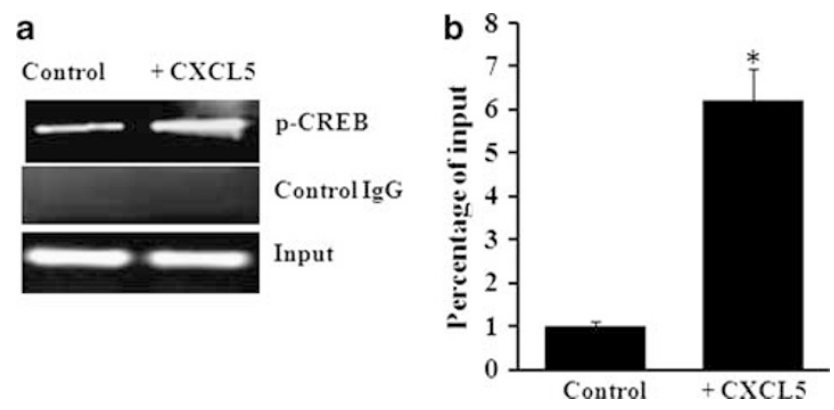

Figure 4 ChIP assay for $p$-CREB-binding to the hRANKL gene promoter region. (a) Normal human bone marrow-derived SAKA-T stromal cells were stimulated with and without CXCL5 $(50 \mathrm{ng} / \mathrm{ml})$ for $24 \mathrm{~h}$, and a ChIP assay was performed using anti-p-CREB antibody as described in the methods. (b). Chromatin immunoprecipitate obtained in the ChIP assay was quantified by real-time PCR. Data represent triplicate studies and are shown as mean \pm s.d. $\left({ }^{*} P<0.05\right)$.

obtained were measured for luciferase activity. As shown in Figure 5a, inhibition of p-CREB using H89 significantly decreased CXCL5-stimulated hRANKL gene promoter activity. Western blot analysis further confirmed H89 inhibition of CXCL5-induced p-CREB expression in SAKA-T cells. We observe a maximum increase in p-CREB expression at 1 hour and a decline to baseline level at the 4-hour period. PKA inhibitor (H89) treatment inhibits p-CREB expression at both time points (Figure $5 \mathrm{~b}$ ). We further demonstrate that inhibitors of p38 MAPK (SB203580), ERK1/2 (PD98059) signaling suppress p-CREB expression and CXCL5 stimulated hRANKL gene promoter activity in these cells (Figures $5 \mathrm{c}$ and $\mathrm{d}$ ). We next examined the CXCL5 effects on osteoblast marker gene expression in human bone marrow-derived stromal/preosteoblast cells. Total RNA isolated from cells stimulated with CXCL5 (50 ng/ml) for $0-48 \mathrm{~h}$ was subjected to RT-PCR analysis for osteoblast marker gene expression. As shown in Figure 6, CXCL5 modulates osteocalcin, collagen type 1 (Col1A) and alkaline phosphatase (ALP) mRNA expressions; however, no significant effect was observed on osterix (Ostx), osteopontin (OPN) and osteoprotegerin (OPG) gene expression in these cells. Thus, our results suggest that elevated levels of CXCL5 contribute to enhanced levels of RANKL expression in PDB.

\section{DISCUSSION}

Elevated levels of RANKL expression has an important role in osteoclast development and excess bone resorption activity in PDB. Several osteotropic factors/resorption stimuli including $1,25-(\mathrm{OH})_{2} \mathrm{D}_{3}$, parathyroid hormone (PTH), IL-1 $\beta$, IL-11 and prostaglandin E2 (PGE2) have been shown to upregulate RANKL expression on marrow stromal/osteoblast cells. ${ }^{6,7,29}$ Abnormal OCLs in PDB are characterized by expression of MVNP transcripts and shown to produce high levels of IL- $6 .{ }^{5}$ However, IL-6 increases RANKL expression in murine systems but does not induce RANKL expression in human marrow stromal cells or osteoblasts. ${ }^{30}$ We measured CXCL5 


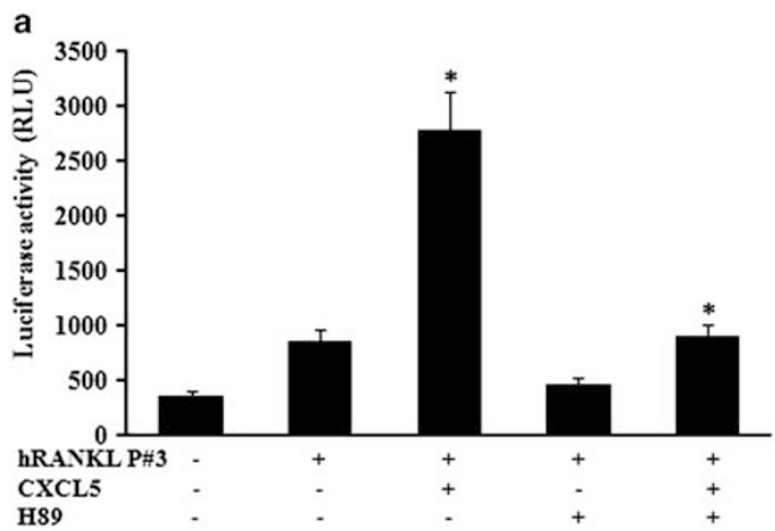

C
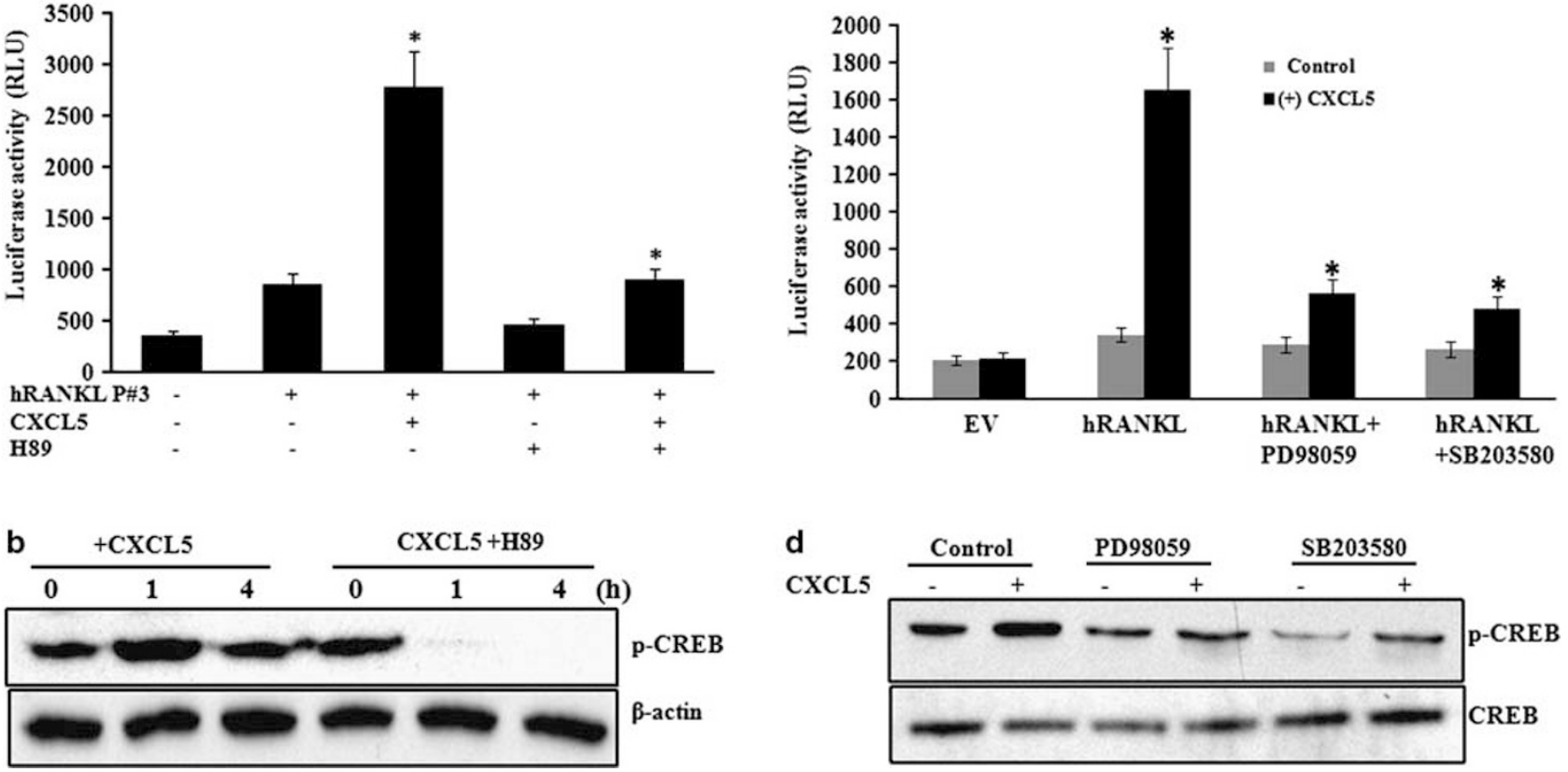

Figure 5 Inhibition of p-CREB suppresses CXCL5-stimulated hRANKL gene promoter activity. (a). Human bone marrow-derived SAKA-T stromal cells were transfected with EV and hRANKL-Luc reporter plasmid (hRANKL P\#3). Cells were stimulated with CXCL5 (50 ng/ml) in the presence and absence of $\mathrm{H} 89(1 \mu \mathrm{M})$ for $48 \mathrm{~h}$. (b). Western blot analysis for H89 inhibition of CXCL5 stimulated p-CREB expression in stromal/preosteoblast cells. (c). Human bone marrow-derived SAKA-T stromal cells were transfected with EV and hRANKL-Luc reporter plasmid (hRANKL P\#3). Cells were stimulated with CXCL5 $(50 \mathrm{ng} / \mathrm{ml})$ in the presence and absence of ERK1/2 activation inhibitor PD98059 $(6.5 \mu \mathrm{M})$ and p38 MAPK inhibitor SB203580 (35 nM) for $48 \mathrm{~h}$. Total cell lysates were assayed for luciferase activity. The transfection efficiency was normalized by $\beta$-galactosidase activity coexpressed in these cells. Data represent triplicate studies and are shown as mean \pm s.d. $\left({ }^{*} P<0.05\right)$. (d). Western blot analysis for ERK1/2 activation inhibitor (PD98059) and p38 inhibitor (SB203580) suppression of CXCL5-stimulated p-CREB expression in stromal/preosteoblast cells.

$(+)$ CXCL5

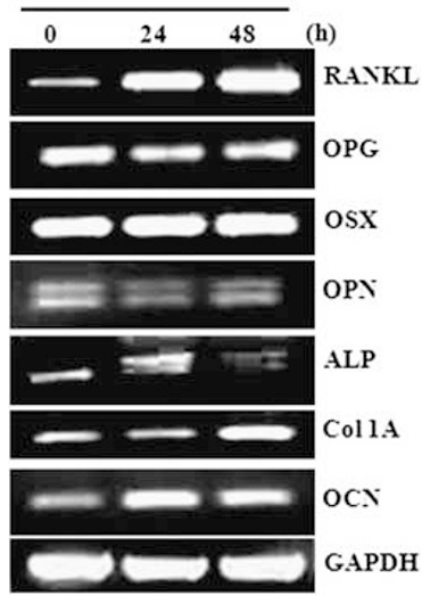

Figure 6 Effect of CXCL5 on osteoblast marker gene expression. Normal human bone marrow-derived stromal/preosteoblast cells were stimulated with CXCL5 $(50 \mathrm{ng} / \mathrm{ml})$ for 24 and $48 \mathrm{~h}$. Total RNA isolated was subjected to RT-PCR analysis for mRNA expression of RANKL, OPG, OSX, OPN, ALP, Col1A and OCN. The relative levels of mRNA expression in the samples were normalized by GAPDH amplification.

levels in serum samples in patients with PDB. Monocytes used to analyze CXCL5 expression were obtained from pagetic bone lesions, which suggest that high levels within the pagetic sites could be detectable at distant marrow sites. Thus the present study demonstrated elevated levels of CXCL5 associated with PDB and a novel function to induce RANKL expression in bone marrow stromal/preosteoblast cells. Our finding that MVNP stimulated CXCL5 production in bone marrow monocytes suggests a potential pathophysiologic role for MVNP expression in PDB. Previously, we have demonstrated that other measles virus genes such as matrix protein does not induce pagetic osteoclast development in bone marrow cultures. ${ }^{4}$ However, MVNP has been shown to induce NF- $\kappa \mathrm{B}$ activation, which may be a potential mechanism in the upregulation of CXCL5 expression in PDB. ${ }^{4,31}$ MVNP has been shown to increase gene expressions of other cytokines such as IL-6, FGF-2 and NFATc1, NF-kB transcription factors that may have both direct and indirect effects on pagetic osteoclast development. Therefore, regulatory mechanisms associated with pagetic osteoclast development are complex. CXCL2 has been shown to enhance the proliferation of bone marrow-derived macrophages through the activation of ERK. Further, RANKL has been shown to induce CXCL2 through JNK and NF- $\kappa \mathrm{B}$ signaling pathways. ${ }^{32}$ Inflammatory cytokines such as IL-1 $\beta$ have been shown to induce CXCL13 production in differentiated osteoblasts, ${ }^{33}$ and human osteoblasts express functional CXCR5 receptors. ${ }^{34}$ Our 
results suggest that CXCL5 regulates both RANKL and CXCR1, but the dependence on CXCR1 of CXCL5-induced RANKL induction is yet to be elucidated. Consistently, we identified CXCL5-activation of p-ERK and p-p38 signaling molecules in bone marrow stromal/preosteoblast cells. Further, the suppression of p-CREB expression by the inhibitors of ERK1/2, p38 and PKA suggests CXCL5 transcriptional control of RANKL gene expression in these cells. CXCL5 showed structural homology with IL-8 (CXCL8), which has been shown to be expressed in PDB. ${ }^{35}$ Therefore, it is possible that IL-8 may modulate RANKL gene expression in stromal/preosteoblast cells. CXCL5 has been shown to promote prostate cancer progression. ${ }^{36}$ Therefore, CXCL5 may have the potential to develop osteosarcoma associated with PDB. It has been reported that Dkk1 that attenuates Wnt3a-dependent inhibition of RANKL in mesenchymal stem cells is upregulated in PDB. ${ }^{37}$ Moreover, methylation of the CpG loci in the RANKL gene promoter has been shown to silence promoter activity. ${ }^{38}$ RANKL signaling generates reactive oxygen species (ROS) in bone marrow macrophage cells. ${ }^{39}$ ROS have been shown to stimulate RANKL gene expression in the bone microenvironment. ${ }^{40}$ Therefore, RANKL expression could be modulated by complex cytokine/chemokine regulatory transcriptional mechanisms in PDB.

Previously, it has been shown that STAT3 activation in stromal/osteoblastic cells is required for RANKL induction by IL-1, but not 1,25-dihydroxyvitamin D3 or PTH. ${ }^{41}$ We recently showed that STAT-1 is the downstream effector of FGF-2 signaling to stimulate RANKL expression in stromal/ preosteoblast cells. ${ }^{13}$ Transcription factors such as Sp1 and Sp3 have been reported to regulate basal RANKL gene transcription in stromal/osteoblast cells. ${ }^{42}$ Mouse RANKL expression has been shown to be upregulated by 1,25 dihydroxyvitamin D3 and PTH through long-range distal enhancer regions. ${ }^{43,44}$ Further, Runx $2 / \mathrm{Cbfa} 1$ has been shown to potentiate PTH stimulation of RANKL through a site in the distal control region. ${ }^{45}$ Recent evidence also suggests that RANKL expression in T cells is regulated by c-Fos through a distal enhancer region. Our findings that the p-CREBbinding motif presents $-2 \mathrm{~kb}$ upstream to the start codon may not exclude the possibility that chemokines may have regulatory motifs in the far upstream sequence $(>2 \mathrm{~kb})$ of the RANKL promoter region. This study identified CXCL5stimulated RANKL expression and that CXCL5 increased p-CREBP binding to the hRANKL promoter region in stromal/preosteoblast cells. Thus, our results suggest that p-CREB is a downstream effector of CXCL5 signaling to induce RANKL expression in bone marrow stromal/ preosteoblast cells and that MVNP-upregulation of CXCL5 contributes to the pathogenesis of PDB.

\section{ACKNOWLEDGEMENTS}

This work was supported by the Department of Defense Medical Research Award PR080480.

\section{DISCLOSURE/CONFLICT OF INTEREST}

The authors declare no conflict of interest.

1. Hansen MF, Seton M, Merchant A. Osteosarcoma in Paget's disease of bone. J Bone and Miner Res 2006;21(Suppl 2):P58-P63.

2. Hocking LJ, Lucas GJ, Daroszewska A, et al. Domain-specific mutations in sequestosome 1 (SQSTM1) cause familial and sporadic Paget's disease. Hum Mol Genet 2002;11:2735-2739.

3. Singer FR, Mills BG. Viruses and Paget's disease. Lancet 1983;1:69.

4. Kurihara N, Reddy SV, Menaa C, et al. Osteoclasts expressing the measles virus nucleocapsid gene display a pagetic phenotype. J Clin Invest 2000;105:607-614.

5. Kurihara N, Zhou H, Reddy SV, et al. Expression of measles virus nucleocapsid protein in osteoclasts induces Paget's disease-like bone lesions in mice. J Bone Miner Res 2006:21:446-455.

6. Nakashima T, Kobayashi $Y$, Yamasaki $S$, et al. Protein expression and functional difference of membrane-bound and soluble receptor activator of NF-kappaB ligand: modulation of the expression by osteotropic factors and cytokines. Biochem Biophys Res Commun 2000;275:768-775.

7. Lee SK, Kalinowski J, Jastrzebski S, et al. 1,25(OH)2 vitamin D3stimulated osteoclast formation in spleen-osteoblast cocultures is mediated in part by enhanced IL-1 alpha and receptor activator of NF-kappa B ligand production in osteoblasts. J Immunol 2002;169: 2374-2380.

8. Rossa C, Ehmann K, Liu M, et al. MKK3/6-p38 MAPK signaling is required for IL-1beta and TNF-alpha-induced RANKL expression in bone marrow stromal cells. J Interferon Cytokine Res 2006;26:719-729.

9. Menaa C, Reddy SV, Kurihara N, et al. Enhanced RANK ligand expression and responsivity of bone marrow cells in Paget's disease of bone. J Clin Invest 2000;105:1833-1838.

10. Sun SG, Lau YS, Itonaga I, et al. Bone stromal cells in pagetic bone and Paget's sarcoma express RANKL and support human osteoclast formation. J Pathol 2006;209:114-120.

11. Neale SD, Schulze E, Smith R, et al. The influence of serum cytokines and growth factors on osteoclast formation in Paget's disease. QJM 2002;95:233-240.

12. Roodman GD, Kurihara N, Ohsaki $\mathrm{Y}$, et al. Interleukin 6. A potential autocrine/paracrine factor in Paget's disease of bone. J Clin Invest 1992;89:46-52.

13. Sundaram K, Senn J, Yuvaraj S, et al. FGF-2 stimulation of RANK ligand expression in Paget's disease of bone. Mol Endocrinol 2009;23: $1445-1454$.

14. Belperio JA, Keane MP, Arenberg DA, et al. CXC chemokines in angiogenesis. J Leukocyte Biol 2000;68:1-8.

15. Zlotnik A, Yoshie O. Chemokines: a new classification system and their role in immunity. Immunity 2000;12:121-127.

16. Walz A, Schmutz $P$, Mueller $C$, et al. Regulation and function of the CXC chemokine ENA-78 in monocytes and its role in disease. J Leukocyte Biol 1997;62:604-611.

17. Li A, King J, Moro A, et al. Overexpression of CXCL5 is associated with poor survival in patients with pancreatic cancer. Am J Pathol 2011;178:1340-1349.

18. Koch $A E$, Kunkel SL, Harlow $L A$, et al. Epithelial neutrophil activating peptide-78: a novel chemotactic cytokine for neutrophils in arthritis. J Clin Invest 1994;94:1012-1018.

19. Sun H, Chung WC, Ryu SH, et al. Cyclic AMP-responsive element binding protein- and nuclear factor-kappaB-regulated CXC chemokine gene expression in lung carcinogenesis. Cancer Prev Res (Phila) 2008; 1:316-328.

20. Wright LM, Maloney $\mathrm{W}, \mathrm{Yu} X$, et al. Stromal cell-derived factor-1 binding to its chemokine receptor CXCR4 on precursor cells promotes the chemotactic recruitment, development and survival of human osteoclasts. Bone 2005;36:840-853.

21. Yu X, Collin-Osdoby P, Osdoby P. SDF-1 increases recruitment of osteoclast precursors by upregulation of matrix metalloproteinase- 9 activity. Connect Tissue Res 2003;44(Suppl 1):79-84.

22. Abe $M$, Hiura $K$, Wilde $J$, et al. Role for macrophage inflammatory protein (MIP)-1alpha and MIP-1beta in the development of osteolytic lesions in multiple myeloma. Blood 2002;100:2195-2202.

23. Sambandam Y, Sundaram K, Liu A, et al. CXCL13 activation of c-Myc induces RANK ligand expression in stromal/preosteoblast cells in the 
oral squamous cell carcinoma tumor-bone microenvironment. Oncogene 2012;32:97-105.

24. Sundaram K, Mani SK, Kitatani K, et al. DACH1 negatively regulates the human RANK ligand gene expression in stromal/preosteoblast cells. J Cell Biochem 2008;103:1747-1759.

25. Roccisana JL, Kawanabe N, Kajiya $\mathrm{H}$, et al. Functional role for heat shock factors in the transcriptional regulation of human RANK ligand gene expression in stromal/osteoblast cells. J Biol Chem 2004;279: 10500-10507.

26. Reddy SV. Etiology of Paget's disease and osteoclast abnormalities. J Cell Biochem 2004;93:688-696.

27. Stadnyk AW, Carrigan SO, Otley AR. Neutrophil transintestinal epithelial migration to CXCR2 ligands is regulated by adenosine. J Pediatr Gastroenterol Nutr 2012;54:414-421.

28. Schauer IE, Knaub LA, Lloyd M, et al. CREB downregulation in vascular disease: a common response to cardiovascular risk. Arterioscler Thromb Vasc Biol 2010;30:733-741.

29. Hofbauer LC, Heufelder AE. Role of receptor activator of nuclear factor-kappaB ligand and osteoprotegerin in bone cell biology. J Mol Med 2001;79:243-253.

30. Hofbauer LC, Khosla S, Dunstan CR, et al. The roles of osteoprotegerin and osteoprotegerin ligand in the paracrine regulation of bone resorption. J Bone Miner Res 2000;15:2-12.

31. Richmond A. Nf-kappa B, chemokine gene transcription and tumour growth. Nat Rev Immunol 2002;2:664-674.

32. Ha J, Choi HS, Lee $Y$, et al. CXC chemokine ligand 2 induced by receptor activator of NF-kappa B ligand enhances osteoclastogenesis. J Immunol 2010;184:4717-4724.

33. Lisignoli G, Cristino S, Toneguzzi S, et al. IL1beta and TNFalpha differently modulate CXCL13 chemokine in stromal cells and osteoblasts isolated from osteoarthritis patients: evidence of changes associated to cell maturation. Exp Gerontol 2004;39:659-665.

34. Lisignoli $G$, Toneguzzi $S$, Piacentini $A$, et al. Human osteoblasts express functional CXC chemokine receptors 3 and 5: activation by their ligands, CXCL10 and CXCL13, significantly induces alkaline phosphatase and beta-N-acetylhexosaminidase release. J Cell Physiol 2003;194:71-79.
35. Birch MA, Ginty AF, Walsh CA, et al. PCR detection of cytokines in normal human and pagetic osteoblast-like cells. J Bone Miner Res 1993:8:1155-1162.

36. Begley LA, Kasina S, Mehra R, et al. CXCL5 promotes prostate cancer progression. Neoplasia 2008;10:244-254.

37. Fujita K, Janz S. Attenuation of WNT signaling by DKK-1 and -2 regulates BMP2-induced osteoblast differentiation and expression of OPG, RANKL and M-CSF. Mol Cancer 2007;6:71.

38. Kitazawa S, Kitazawa R. Epigenetic control of mouse receptor activator of NF-kappa B ligand gene expression. Biochem Biophys Res Commun 2002;293:126-131.

39. Lee NK, Choi YG, Baik JY, et al. A crucial role for reactive oxygen species in RANKL-induced osteoclast differentiation. Blood 2005;106: 852-859.

40. Bai XC, Lu D, Liu AL, et al. Reactive oxygen species stimulates receptor activator of NF-kappaB ligand expression in osteoblast. J Biol Chem 2005;280:17497-17506.

41. O'Brien CA, Gubrij I, Lin SC, et al. STAT3 activation in stromal/ osteoblastic cells is required for induction of the receptor activator of NF-kappaB ligand and stimulation of osteoclastogenesis by gp130utilizing cytokines or interleukin-1 but not 1,25-dihydroxyvitamin D3 or parathyroid hormone. J Biol Chem 1999;274:19301-19308.

42. Liu J, Yang H, Liu W, et al. Sp1 and Sp3 regulate the basal transcription of receptor activator of nuclear factor kappa B ligand gene in osteoblasts and bone marrow stromal cells. J Cell Biochem 2005; 96:716-727.

43. Fu Q, Manolagas SC, O'Brien CA. Parathyroid hormone controls receptor activator of NF-kappaB ligand gene expression via a distant transcriptional enhancer. Mol Cell Biol 2006;26:6453-6468.

44. Kim S, Yamazaki M, Zella LA, et al. Activation of receptor activator of NF-kappaB ligand gene expression by 1,2-dihydroxyvitamin D3 is mediated through multiple long-range enhancers. Mol Cell Biol 2006;26:6469-6486.

45. Onal M, Galli $\mathrm{C}$, Fu Q, et al. The RANKL distal control region is required for the increase in RANKL expression, but not the bone loss, associated with hyperparathyroidism or lactation in adult mice. Mol Endocrinol 2012;26:341-348. 